[8] _ , "Design of multichannel nonuniform transmultiplexers using general building blocks," IEEE Trans. Signal Processing, vol. 49, pp. 91-99, Jan. 2001

[9] R. P. Ramachandran and P. Kabal, "Transmultiplexers: Perfect reconstruction and compensation of channel distortion," Signal Process., vol. 21, pp. 261-274, 1990.

[10] T. D. Tran, " $M$-channel linear phase perfect reconstruction filter bank with rational coefficients," IEEE Trans. Circuits Syst. I, vol. 49, pp. 914-927, July 2002.

[11] P. P. Vaidyanathan, "Theory and design of $M$-channel maximally decimated quadrature mirror filters with arbitrary $M$, having the perfect-reconstruction property," IEEE Trans. Acoust., Speech, Signal Processing, vol. ASSP-35, pp. 476-492, Apr. 1987.

[12] —, Multirate Systems and Filter Banks. Englewood Cliffs, NJ: Prentice-Hall, 1993.

[13] M. Vetterli, "Perfect transmultiplexers," in Proc. IEEE Int. Acoustics, Speech, and Signal Processing Conf., Apr. 1986, pp. 2567-2570.

[14] G. W. Wornell, "Emerging applications of multirate signal processing and wavelets in digital communications," Proc. IEEE, vol. 84, pp. 586-603, Apr. 1996

[15] S.-J. Yang, J.-H. Lee, and B.-C. Chieu, "Perfect-reconstruction filter banks having linear-phase FIR filters with equiripple response," IEEE Trans. Signal Processing, vol. 46, pp. 3246-3255, Dec. 1998.

\section{Stability and Control of Differential Linear Repetitive Processes Using an LMI Setting}

\author{
K. Galkowski, W. Paszke, E. Rogers, S. Xu, J. Lam, and D. H. Owens
}

\begin{abstract}
This paper considers differential linear repetitive processes which are a distinct class of two-dimensional continuous-discrete linear systems of both physical and systems theoretic interest. The substantial new results are on the application of linear-matrix-inequality-based tools to stability analysis and controller design for these processes, where the class of control laws used has a well defined physical basis. It is also shown that these tools extend naturally to cases when there is uncertainty in the state-space model of the underlying dynamics.
\end{abstract}

Index Terms-Controller design, linear matrix inequality (LMI) design, repetitive dynamics, uncertainty.

\section{INTRODUCTION}

The essential unique characteristic of a repetitive, or multipass, process is a series of sweeps-termed passes - through a set of dynamics defined over a fixed finite duration known as the pass length. On each pass, an output-termed the pass profile-is produced which acts as a forcing function on, and hence contributes to, the dynamics of the next pass profile. This, in turn, leads to the unique control problem for these processes in that the output sequence of pass

Manuscript received December 8, 2002; revised February 21, 2003. Please note that the review process for this paper was handled exclusively by the TCAS-I editorial office and that the paper has been published in TCAS-II for logistical reasons. This paper was recommended by Associate Editor C. Xiao.

K. Galkowski and W. Paszke are with the Institute of Control and Computation Engineering, University of Zielona Gora, Zielona Gora, Poland.

E. Rogers is with the Department of Electronics and Computer Science, University of Southampton, Southampton SO17 1BJ, U.K

$\mathrm{S}$. Xu and J. Lam are with the Department of Mechanical Engineering, University of Hong Kong, Kowloon, Hong Kong.

D. H. Owens is with the Department of Automatic Control and Systems Engineering, University of Sheffield, Sheffield S10 2TN, U.K.

Digital Object Identifier 10.1109/TCSII.2003.816909 profiles generated can contain oscillations that increase in amplitude in the pass-to-pass direction.

To introduce a formal definition, let $\alpha<+\infty$ denote the pass length (assumed constant). Then in a repetitive process the pass profile $y_{k}(t)$, $0 \leq t \leq \alpha$, generated on pass $k$ acts as a forcing function on, and hence contributes to, the dynamics of the next pass profile $y_{k+1}(t)$, $0 \leq t \leq \alpha, k \geq 0$.

Physical examples of repetitive processes include long-wall coal cutting and metal rolling operations; see, for example, [1]. Also, in recent years, applications have arisen where adopting a repetitive process setting for analysis has distinct advantages over alternatives. Examples of these so-called algorithmic applications of repetitive processes include classes of iterative learning control (ILC) schemes [5] and iterative algorithms for solving nonlinear dynamic optimal control problems based on the maximum principle [6]. In the case of ILC for the linear dynamics case, the stability theory for differential and discrete linear repetitive processes is the essential basis for a rigorous stability/convergence analysis of such schemes.

Attempts to control these processes using standard, termed one-dimensional (1-D) here, systems theory/algorithms fail (except in a few very restrictive special cases) precisely because such an approach ignores their inherent two-dimensional (2-D) systems structure, i.e., information propagation occurs from pass-to-pass and along a given pass, and also the effects of resetting the pass initial conditions before the start of each new pass. A rigorous stability theory for linear repetitive processes has been developed. This theory [7] is based on an abstract model in a Banach space setting which includes all such processes as special cases. Also, the results of applying this theory to a wide range of such cases have been reported [1], [7]. These include the processes considered here, where the resulting conditions can, as one alternative, be tested by direct application of well-known 1-D linear systems stability tests.

One unique feature of repetitive processes is that it is possible define physically meaningful control laws for them. For example, in the ILC application, one such family of control laws is composed of, state or output based, feedback control action on the current pass combined with information "fed forward" from the previous pass, or trial in the ILC context, which, of course, has already been generated and is therefore available for use. It is, hence, highly desirable to have an analysis setting where such control laws can be designed for stability and/or guaranteed performance.

In this paper, we show that a linear matrix inequality (LMI) reformulation (see [2] for the background) of the stability condition leads naturally to design algorithms for control laws of the type discussed above. It is also shown that this setting enables some significant progress to be made on the currently essentially open problem of the stability and control of these processes is the presence of uncertainty in the model structure.

\section{BACKGROUND AND LMI STABILITY ANALYSIS}

The state-space model of the differential linear repetitive processes considered here has the following form over $0 \leq t \leq \alpha, k \geq 0$ :

$$
\begin{aligned}
& \dot{x}_{k+1}(t)=A x_{k+1}(t)+B u_{k+1}(t)+B_{0} y_{k}(t) \\
& y_{k+1}(t)=C x_{k+1}(t)+D u_{k+1}(t)+D_{0} y_{k}(t)
\end{aligned} .
$$

Here, on pass $k, x_{k}(t)$ is the $n \times 1$ state vector, $y_{k}(t)$ is the $m \times 1$ pass-profile vector, and $u_{k}(t)$ is the $l \times 1$ vector of control inputs.

To complete the process description, it is necessary to specify the "initial conditions" - termed the boundary conditions here, i.e., the state initial vector on each pass and the initial pass profile. Here, no 
loss of generality arises from assuming $x_{k+1}(0)=d_{k+1}, k \geq 0$, and $y_{0}(t)=f(t)$, where $d_{k+1}$ is an $n \times 1$ vector with known constant entries and $f(t)$ is an $m \times 1$ vector whose entries are known functions of $t$. For ease of presentation, no further explicit reference will be made to the boundary conditions.

The stability theory for a linear-constant pass-length repetitive processes consists of two distinct concepts, but here it is the stronger of these which is required. This is termed stability along the pass and several equivalent sets of necessary and sufficient conditions for processes described by (1) to have this property are known [7], but here it is the sufficient condition of Theorem 1 below which will be used in this work. A central feature of the results here is that they will show that this sufficient condition allows us to design control laws in a straightforward manner, whereas the currently available necessary and sufficient conditions only really allow us to obtain conditions for stability under control action.

In this paper, $>0(<0)$ denotes a symmetric positive (negative) definite matrix.

Theorem 1: Differential linear repetitive processes described by a state-space model of the form (1) are stable along the pass if there exist matrices $W=W_{1} \oplus W_{2}>0$ and $Q>0$ which solve the following so-called the 2-D Lyapunov equation:

$$
\widehat{A}^{T} W^{1,0}+W^{1,0} \widehat{A}+\widehat{A}^{T} W^{0,1} \widehat{A}-W^{0,1}=-Q<0
$$

where $\oplus$ denotes the direct sum, i.e., $W=\operatorname{diag}\left\{W_{1}, W_{2}\right\}, 0_{p}$ the null matrix of dimension $p \times p$, and

$$
\widehat{A}=\left[\begin{array}{cc}
A & B_{0} \\
C & D_{0}
\end{array}\right], W^{1,0}=W_{1} \oplus 0_{m}, W^{0,1}=0_{n} \oplus W_{2} .
$$

This result was established for differential linear repetitive processes in [7] but it has its origins in work on delay differential systems (see the relevant references in [7]). Also, in the single-input single-output (SISO) case, it has been shown [3] that this 2-D Lyapunov equation condition is both necessary and sufficient for stability along the pass.

The 2-D Lyapunov (2) can be rewritten in the form

$$
\widehat{A}_{2}^{T} \widehat{W}_{2} \widehat{A}_{2}-W^{0,1}+\widehat{A}_{1}^{T} W^{1,0}+W^{1,0} \widehat{A}_{1}<0
$$

where $\widehat{W}_{2}=W_{3} \oplus W_{2}$, the $n \times n$ matrix $W_{3}>0$ is arbitrary, and

$$
\widehat{A_{1}}=\left[\begin{array}{cc}
A & B_{0} \\
0 & 0
\end{array}\right], \quad \widehat{A}_{2}=\left[\begin{array}{cc}
0 & 0 \\
C & D_{0}
\end{array}\right] \text {. }
$$

Now apply the well-known Schur complements formula to (3) with $W=-W^{0,1}+\widehat{A}_{1}^{T} W^{1,0}+W^{1,0} \widehat{A}_{1}, L=\widehat{A}_{2}$ and $V=\widehat{W}_{2}$, and then left and right multiply the result by the matrix $\left[I \oplus \widehat{W}_{2}\right]$ to obtain the equivalent condition

$$
\left[\begin{array}{cc}
-W^{0,1}+\widehat{A}_{1}^{T} W^{1,0}+W^{1,0} \widehat{A}_{1} & \widehat{A}_{2}^{T} \widehat{W}_{2} \\
\widehat{W}_{2} \widehat{A}_{2} & -\widehat{W}_{2}
\end{array}\right]<0 .
$$

This is clearly in LMI form, and hence we immediately have the following result.

Theorem 2: Differential linear repetitive processes described by a state-space model of the form (1) are stable along the pass if the LMI of (5) is feasible.

\section{LMI-BASED CONTROLLER DESIGN}

The design of control laws for 2-D discrete linear systems described by the Roesser and Fornasini Marchesini state-space models (see, for example, the relevant references in [7]) has received considerable attention in the literature over the years. A valid criticism of such work, however, is that the structure of the control algorithms are not well founded physically. In particular, for a 1-D linear system with state $x(t)$ and input $u(t)$, a state feedback control law with zero tracking vector is of the form $u(t)=F x(t)$ but in the 2-D case the natural generalization could replace $x(t)$ with either the local or global state vector. Also in the absence of generalizations of well-defined and understood 1-D concepts, e.g., the pole assignment problem and error-actuated output feedback control action, it has not been really possible to formulate a control design problem beyond that of obtaining conditions for stabilization under the control action for the case of a model which, in effect, is assumed to be an exact representation of the underlying dynamics.

The first difficulty above does not arise with differential linear repetitive processes as discussed below. Also, it is shown in the next section that the controller design is possible in an LMI setting which also extends to cases where there is uncertainty in the model structure.

In the case of repetitive processes, it is physically meaningful to define the current pass output error as the difference, at each point along the pass, between a specified reference trajectory for that pass, which in most cases will be the same on each pass, and the actual pass-profile produced. Then we can define a so-called current pass error actuated controller which uses the generated error vector to construct the current pass control input vector. Preliminary work (e.g., [1]) has shown that, except in a few very restrictive special cases, the controller used must be actuated by a combination of current pass information and "feedforward" information from the previous pass to guarantee even stability along the pass closed loop. Note here also that in the ILC application area, the previous pass (or trial in the ILC setting) output vector is an obvious signal to use as "feedforward" action.

As the first major systematic attempt at controller design for differential linear repetitive processes, we consider a control law of the following form over $0 \leq t \leq \alpha, k \geq 0$ :

$$
u_{k+1}(t)=K_{1} x_{k+1}(t)+K_{2} y_{k}(t):=K\left[\begin{array}{c}
x_{k+1}(t) \\
y_{k}(t)
\end{array}\right] .
$$

In effect, this control law uses feedback of the current pass state vector, which is assumed to be available for use here, and "feedforward" of the previous pass-profile vector.

This control law has clear physical meaning for practical applications of differential linear repetitive processes and the obvious question now to ask is: can we obtain conditions for closed loop stability along the pass coupled with easily, in relative terms, applied algorithms for computing the controller parameters? Here, we show that the answer to this question is yes if the LMI setting of the previous section is used. Note that there may well be alternative approaches to this question-if this is indeed the case, then the results here will serve as a baseline comparison.

Application of the control law (6) to (1) and applying a known result from [7]gives the following necessary and sufficient condition for closed-loop stability along the pass:

$$
C_{c}(s, z) \neq 0, \forall(s, z) \operatorname{Re}(s) \geq 0,|z| \leq 1
$$

where

$$
C_{c}(s, z)=\operatorname{det}\left[s I_{n} \oplus I_{m}-\left[\begin{array}{cc}
\left(A+B K_{1}\right) & \left(B_{0}+B K_{2}\right) \\
z\left(C+D K_{1}\right) & z\left(D_{0}+D K_{2}\right)
\end{array}\right]\right] \text {. }
$$

Now introduce the matrices

$$
\widehat{B}_{1}=\left[\begin{array}{l}
B \\
0
\end{array}\right], \quad \widehat{B}_{2}=\left[\begin{array}{l}
0 \\
D
\end{array}\right] .
$$

Then, we have the following theorem which results directly from interpreting (5) closed loop.

Theorem 3: Consider a differential linear repetitive process with state-space model of the form (1) subject to a control law of the form (6). Then the resulting closed-loop process is stable along the pass if 
there exist matrices $Y>0, Z>0$ together with matrices $N$ and $M$, such that the following LMI holds:

$$
\left[\begin{array}{ccc}
Y A^{T}+N^{T} B^{T}+A Y+B N & B_{0} Z+B M & Y C^{T}+N^{T} D^{T} \\
Z B_{0}^{T}+M^{T} B^{T} & -Z & Z D_{0}^{T}+M^{T} D^{T} \\
C Y+D N & D_{0} Z+D M & -Z
\end{array}\right]<0 .
$$

Also, if (10) holds, stabilizing $K_{1}$ and $K_{2}$ for the control law (6) are given by

$$
K_{1}=N Y^{-1}, K_{2}=M Z^{-1} .
$$

Proof: Based on (5) and (6), the closed-loop system is stable along the pass if there exist symmetric matrices $W_{1}>0$ and $W_{2}>0$, such that we get (12), shown at the bottom of the page. The difficulty with the matrix inequality (12) is that it is nonlinear in its parameters. To obtain a strict LMI form first note that the above condition can be rewritten, by direct substitution for the relevant submatrices, such as (13), shown at the bottom of the page. Next, pre- and post-multiply (13) by the matrix $\operatorname{diag}\left[W_{1}^{-1}, W_{2}^{-1}, W_{2}^{-1}, W_{2}^{-1}\right]$ and then note that the third block row and the third block column of the resulting matrix can be removed without changing the underlying inequality solution. Now introduce the substitutions $Y=W_{1}^{-1}$ and $Z=W_{2}^{-1}$, and use (11) to obtain (10).

\section{STABILITY AND CONTROL OF UNCERTAIN DIFFERENTIAL LiNEAR REPETITIVE PROCESSES}

One key area for which no results are currently available is the stability and control of differential linear repetitive processes in the presence of uncertainty in the model structure. Also, a natural place to begin work in this area is to impose uncertainty structures on the matrices which define the state-space model of the process under consideration. In this section, we show that the LMI-based stability and controller design analysis given in the previous section can be extended to two cases where the uncertainty is so expressed.

In the case of both 1-D discrete and differential linear systems, commonly used uncertainty descriptions for robust control studies are either norm bounded or of a polytopic form; see, for example, [4] and the relevant references cites in this paper. Both of these representations have their advantages and disadvantages and also there are practically relevant problem areas where one is more suitable than the other. Note also that differential linear repetitive processes, and their discrete counterparts, have quite strong structural links with 1-D differential and discrete linear systems.

For the processes considered here, the links with 1-D differential linear systems are associated with the structure of the linear differential equation governing the along the pass state dynamics and that with 1-D discrete linear systems from the updating of the pass-profile vector from pass-to-pass. Based on this, the rest of this section considers two cases, where in the first one the uncertainty in the current pass state dynamics updating has a polytopic form and the pass-to-pass updating uncertainty is norm bounded. In the second case, both uncertainty structures are assumed to be norm bounded.

Case A: Here, we assume that the uncertainty in the differential equation which governs the evolution of the current pass state vector equation in (1) has a polytopic character. In particular, it is assumed that all possible choices for the matrices which define this linear matrix differential equation can be expressed as

$$
\left[\begin{array}{lll}
A & B & B_{0}
\end{array}\right] \in \operatorname{Co}\left[\begin{array}{lll}
A^{i} & B^{i} & B_{0}^{i}
\end{array}\right], \quad i=1,2, \ldots, h
$$

and (15), shown at the bottom of the page, where $A^{i}, B_{0}^{i}$, and $B^{i}$ are vertex matrices of the required dimensions with known constant entries.

For the current pass-profile updating equation in (1) we assume a standard norm-bound on the perturbations, i.e., this equation is replaced by

$$
\begin{aligned}
y_{k+1}(t)=(C+\Delta C) x_{k+1}(t) \\
+(D+\Delta D) u_{k+1}(t)+\left(D_{0}+\Delta D_{0}\right) y_{k}(t)
\end{aligned}
$$

where

$$
\left[\begin{array}{ccc}
\Delta C & \Delta D_{0} & \Delta D
\end{array}\right]=H F\left[\begin{array}{lll}
E_{1}^{1} & E_{1}^{2} & E_{2}
\end{array}\right]
$$

and

$$
F^{T} F \leq I
$$

where $H, E_{1}^{1}, E_{1}^{2}$, and $E_{2}$ are matrices of compatible dimensions whose entries are known. The $F$ has constant entries of unknown values which must be determined from knowledge available about the actual process under consideration.

Case B: Here, we assume a norm-bounded perturbation in both the current pass state and pass-profile updating equations and, in particular, the latter is as in Case A above and the former takes the form

$$
\begin{aligned}
\dot{x}_{k+1}(t)=(A+\Delta A) x_{k+1}(t)+(B+\Delta B) u_{k+1}(t) \\
+\left(B_{0}+\Delta B_{0}\right) y_{k}(t)
\end{aligned}
$$

where

$$
\left[\begin{array}{lll}
\Delta A & \Delta B_{0} & \Delta B \\
\Delta C & \Delta D_{0} & \Delta D
\end{array}\right]=\left[\begin{array}{l}
H_{1} \\
H_{2}
\end{array}\right] F\left[\begin{array}{lll}
E_{1}^{1} & E_{1}^{2} & E_{2}
\end{array}\right]
$$

and (18) is again assumed to hold.

Consider now Case A. Then the following two results develop an LMI sufficient condition for stability along the pass in this case.

$$
\left[\begin{array}{cc}
-W^{0,1}+\left(\widehat{A}_{1}+\widehat{B}_{1} K\right)^{T} W^{1,0}+W^{1,0}\left(\widehat{A}_{1}+\widehat{B}_{1} K\right) & \left(\widehat{A}_{2}+\widehat{B}_{2} K\right)^{T} \widehat{W}_{2} \\
\widehat{W}_{2}\left(\widehat{A}_{2}+\widehat{B}_{2} K\right) & -\widehat{W}_{2}
\end{array}\right]<0
$$

$$
\left[\begin{array}{cccc}
\left(A+B K_{1}\right)^{T} W_{1}+W_{1}\left(A+B K_{1}\right) & W_{1}\left(B_{0}+B K_{2}\right) & 0 & \left(C+D K_{1}\right)^{T} W_{2} \\
\left(B_{0}+B K_{2}\right)^{T} W_{1} & -W_{2} & 0 & \left(D_{0}+D K_{2}\right)^{T} W_{2} \\
0 & 0 & -W_{2} & 0 \\
W_{2}\left(C+D K_{1}\right) & W_{2}\left(D_{0}+D K_{2}\right) & 0 & -W_{2}
\end{array}\right]<0
$$

$$
\operatorname{Co}\left[\begin{array}{lll}
A^{i} & B_{0}^{i} & B^{i}
\end{array}\right]:=\left\{X: X=\sum_{i=1}^{h} \alpha_{i}\left[A^{i}, \quad B_{0}^{i}, \quad B^{i}\right], \quad \alpha_{i} \geq 0, \quad \sum_{i=1}^{h} \alpha_{i}=1\right\}
$$


Theorem 4: Consider a differential linear repetitive process whose state-space model is of the form of Case A above. Then this process is stable along the pass if there exist matrices $W_{1}>0$ and $W_{2}>0$, which satisfy the following set of LMIs of the generalized Lyapunov type:

$$
\begin{array}{r}
\widehat{A}_{1}^{i T} W^{1,0}+W^{1,0} \widehat{A}_{1}^{i}-W^{0,1}+\left(\widehat{A}_{2}+\widetilde{H} \widehat{F} \widehat{E}_{1}\right)^{T} \widehat{W}_{2}\left(\widehat{A}_{2}+\widetilde{H} \widehat{F} \widehat{E}_{1}\right) \\
<0, \quad i=1,2, \ldots, h \quad(21)
\end{array}
$$

where $\widehat{A}_{2}$ is given by (4) and

$$
\begin{aligned}
\widehat{A}_{1}^{i} & =\left[\begin{array}{cc}
A^{i} & B_{0}^{i} \\
0 & 0
\end{array}\right], \widetilde{H}=\left[\begin{array}{cc}
0 & 0 \\
H & H
\end{array}\right] \\
\widehat{F} & =\left[\begin{array}{cc}
F & 0 \\
0 & F
\end{array}\right], \widehat{E}_{1}=\left[\begin{array}{cc}
E_{1}^{1} & 0 \\
0 & E_{1}^{2}
\end{array}\right]
\end{aligned}
$$

Proof: This follows immediately on use of the easily established fact that a stability property for the polytopic uncertainty considered here holds provided it also holds for each "vertex" model $A^{i}, B_{0}^{i}, B^{i}$, $C, D_{0}$, and $D$.

Once again, this result is difficult to apply since the underlying matrix inequality still contains an uncertainty matrix $F$ and is not in exact LMI form. To avoid these problems, we make use of the following well-known result [4] (interpreted in terms of the current analysis).

Lemma 1: The conditions of (21) hold if, and only if, there exists a scalar $\epsilon>0$ and matrices $W_{1}>0$ and $W_{2}>0$, such that for $i=1,2, \ldots, h$, we have (23), shown at the bottom of the page.

Now we can establish the following result which is in the desired LMI form.

Theorem 5: Consider a differential linear repetitive process whose state-space model is of the form of Case A above. Then this process is stable along the pass if there exist matrices $W_{1}>0$ and $W_{2}>0$ and a real scalar $\mu>0$, such that the following set of LMIs is feasible for $i=1,2, \ldots, h$ :

$$
\left[\begin{array}{cccc}
-\widehat{W}_{2} & \widehat{W}_{2} \widehat{A}_{2} & \widehat{W}_{2} \widetilde{H} & 0 \\
\widehat{A}_{2}^{T} \widehat{W}_{2} & \widehat{A}_{1}^{i T} W^{1,0}+W^{1,0} \widehat{A}_{1}^{i}-W^{0,1} & 0 & \mu \widehat{E}_{1}^{T} \\
\widetilde{H}^{T} \widehat{W}_{2} & 0 & -\mu I & 0 \\
0 & \mu \widehat{E}_{1} & 0 & -\mu I
\end{array}\right]<0 .
$$

Proof: First apply Schur's complement formula to (23) with

$$
\begin{aligned}
W & =\left[\begin{array}{cc}
-\widehat{W}_{2} & \widehat{W}_{2} \widehat{A}_{2} \\
\widehat{A}_{2}^{T} \widehat{W}_{2} & \widehat{A}_{1}^{i T} W^{1,0}+W^{1,0} \widehat{A}_{1}^{i}-W^{0,1}
\end{array}\right] \\
V & =\left[\begin{array}{cc}
\epsilon I & 0 \\
0 & \epsilon^{-1} I
\end{array}\right], \quad L=\left[\begin{array}{cc}
\widetilde{H}^{T} & 0 \\
0 & \widehat{E}_{1}
\end{array}\right] .
\end{aligned}
$$

Finally, left and right multiply the result of this last step by $\operatorname{diag}\left[\widehat{W}_{2}, \quad I, \quad I, \epsilon^{-1} I\right]$, and introduce the change of variable $\epsilon^{-1}:=\mu$ to obtain (24).
To begin consideration of Case $\mathrm{B}$, introduce the notation

$$
\Delta \widehat{A}_{1}=\left[\begin{array}{cc}
\Delta A & \Delta B_{0} \\
0 & 0
\end{array}\right]=\left[\begin{array}{c}
H_{1} \\
0
\end{array}\right] F\left[E_{1}^{1} E_{1}^{2}\right]=\widehat{H}_{1} F \widehat{E}
$$

and

$$
\Delta \widehat{A}_{2}=\left[\begin{array}{cc}
0 & 0 \\
\Delta C & \Delta D_{0}
\end{array}\right]=\left[\begin{array}{c}
0 \\
H_{2}
\end{array}\right] F\left[\begin{array}{ll}
E_{1}^{1} & E_{1}^{2}
\end{array}\right]=\widehat{H}_{2} F \widehat{E} .
$$

Also, we will use the following result.

Lemma 2: [4] Let $\Sigma_{1}, \Sigma_{2}$ and $\Delta$ be real matrices of appropriate dimensions. Then for any $\Delta^{T} \Delta \leq I$ and scalar $\epsilon>0$ the following inequality is satisfied:

$$
\Sigma_{1} \Delta \Sigma_{2}+\Sigma_{2}^{T} \Delta \Sigma_{1}^{T} \leq \epsilon^{-1} \Sigma_{1} \Sigma_{1}^{T}+\epsilon \Sigma_{2}^{T} \Sigma_{2} .
$$

Theorem 6: Consider a differential linear repetitive process whose state-space model is of the form of Case B above. Then this process is stable along the pass if there exist scalars $\epsilon_{1}$ and $\epsilon_{2}>0$ and matrices $W_{1}>0, W_{2}>0$, and $W_{3}>0$, such that the LMI in (28) is feasible, as shown at the bottom of the page.

Proof: It follows immediately from Theorem 2 that stability along the pass in this case holds if we have (29), shown at the bottom of the page. which can be easily decomposed into

$$
\begin{aligned}
& {\left[\begin{array}{cc}
-W^{0,1}+\widehat{A}_{1}^{T} W^{1,0}+W^{1,0} \widehat{A}_{1} & \widehat{A}_{2}^{T} \widehat{W}_{2} \\
\widehat{W}_{2} \widehat{A}_{2} & -\widehat{W}_{2}
\end{array}\right]} \\
& +\left[\begin{array}{cc}
\Delta \widehat{A}_{1}^{T} W^{1,0}+W^{1,0} \Delta \widehat{A}_{1} & \Delta \widehat{A}_{2}^{T} \widehat{W}_{2} \\
\widehat{W}_{2} \Delta \widehat{A}_{2} & 0
\end{array}\right]<0 .
\end{aligned}
$$

Using (27), we now have that

$$
\left[\begin{array}{cc}
\Delta \widehat{A}_{1}^{T} W^{1,0}+W^{1,0} \Delta \widehat{A}_{1} & \Delta \widehat{A}_{2}^{T} \widehat{W}_{2} \\
\widehat{W}_{2} \Delta \widehat{A}_{2} & 0
\end{array}\right] \leq\left[\begin{array}{cc}
\Lambda+\epsilon_{1} E^{T} E & 0 \\
0 & \epsilon_{2} \widehat{E}^{T} \widehat{E}
\end{array}\right]
$$

where

$$
\Lambda=\epsilon_{1}^{-1} W^{1,0} \widehat{H}_{1} \widehat{H}_{1}^{T} W^{1,0}+\epsilon_{2}^{-1} \widehat{W}_{2} \widehat{H}_{2} \widehat{H}_{2}^{T} \widehat{W}_{2} .
$$

The proof is now completed by an obvious use of the Schur complement and an equally obvious congruence transform.

Suppose now that a process from either Case A or Class B is subject to a control law of the form (6). Then it is straightforward to show that the results established in this section can also be applied to the resulting state-space model to give a (sufficient) condition for closed-loop stability along the pass and (as in the previous section) a formula for designing the corresponding control law. Hence, the details are omitted here. One point to note, however, is that the LMIs in this analysis could well be "large" and hence the possibility of numerical difficulties; this is left here as an area for further research. Note also that the results given here are for uncertainty matrices with constant entries but extend

$$
\begin{gathered}
{\left[\begin{array}{ccc}
-\widehat{W}_{2}^{-1}+\epsilon \widetilde{H} \widetilde{H}^{T} & \widehat{A}_{2} \\
\widehat{A}_{2}^{T} & \epsilon^{-1} \widehat{E}_{1}^{T} \widehat{E}_{1}+\widehat{A}_{1}^{i T} W^{1,0}+W^{1,0} \widehat{A}_{1}^{i}-W^{0,1}
\end{array}\right]<0} \\
{\left[\begin{array}{cccc}
-W^{0,1}+\widehat{A}_{1}^{T} W^{1,0}+W^{1,0} \widehat{A}_{1}+\epsilon_{1} \widehat{E}^{T} \widehat{E} & \widehat{A}_{2}^{T} \widehat{W}_{2} & W^{1,0} \widehat{H}_{1} & \widehat{W}_{2} \widehat{H}_{2} \\
\widehat{W}_{2} \widehat{A}_{2} & -\widehat{W}_{2}+\epsilon_{2} \widehat{E}^{T} \widehat{E} & 0 & 0 \\
\widehat{H}_{1}^{T} W^{1,0} & 0 & -\epsilon_{1} I & 0 \\
\widehat{H}_{2}^{T} \widehat{W}_{2} & 0 & 0 & -\epsilon_{2} I
\end{array}\right]<0}
\end{gathered}
$$

$$
\left[\begin{array}{cc}
-W^{0,1}+\left(\widehat{A}_{1}+\Delta \widehat{A}_{1}\right)^{T} W^{1,0}+W^{1,0}\left(\widehat{A}_{1}+\Delta \widehat{A}_{1}\right) & \left(\widehat{A}_{2}+\Delta \widehat{A}_{2}\right)^{T} \widehat{W}_{2} \\
\widehat{W}_{2}\left(\widehat{A}_{2}+\Delta \widehat{A}_{2}\right) & -\widehat{W}_{2}
\end{array}\right]<0
$$


in a natural manner to the case when they are functions of $t$, where $F(t)$ and $\Delta(t)$ must satisfy (18) for all $t$.

\section{CONCLUSIONS}

Differential linear repetitive processes are a distinct class of 2-D continuous-discrete linear systems of both applications and systems theoretic interest. In applications, they arise in ILC schemes and in solution algorithms for nonlinear dynamic optimal control algorithms based on the maximum principle. Repetitive processes cannot be analyzed/controlled by direct application of existing systems theory and currently there is only a very limited literature on the specification and design of control schemes for them and essentially none on the class of processes considered in this paper.

The most significant new contribution in this paper is that an LMI formulation of stability along the pass (the stronger form of the two distinct stability concepts for these processes which will most often be required in applications) can be immediately used to design a powerful class of control laws for these processes which, crucially, have a well defined physical interpretation for applications areas such as ILC. These features are missing from alternative stability characterizations where the most that can be achieved is to test the resulting conditions using 1-D linear systems stability tests.

It is important to place the results of this paper in context; essentially, they represent the first systematic procedure for stability analysis and onward controller design, as opposed to just stability analysis only, for a very important and distinct class of 2-D linear systems using control laws which are well grounded in terms of the underlying process dynamics. One key area for which no results are currently available is the stability and control of differential linear repetitive processes in the presence of uncertainty in the model structure. Here, it has been shown that the LMI setting immediately allows significant progress to be made.

One counter argument here may be that the uncertainty structures used here are well known in the 1-D linear systems area. This is, in fact, true, but only in terms of some of the matrices in the defining repetitive process state-space model but, given the facts that: 1) no previous work has been done in this area and 2) these processes do have certain structural similarities with 1-D differential and discrete linear systems, this is not an unreasonable place to begin work. The most important conclusion to be drawn is, we argue, that it is indeed possible to control these processes in the presence of uncertainty in the defining model structure and that the results so obtained provide a useful benchmark for further work. Also, the numerics associated with the resulting conditions may not always be well behaved and this area also merits further attention.

\section{REFERENCES}

[1] S. E. Benton, "Analysis and Control of Linear Repetitive Processes," Ph.D. dissertation, Univ. Southampton, Southampton, U.K., 2000.

[2] S. Boyd, L. E. Ghaoui, E. Feron, and V. Balakrishnan, "Linear Matrix Inequalities in Systems and Control Theory," in SIAM Studies in Applied Mathematics. Philadelphia, PA: SIAM.

[3] S. Foda and P. Agathoklis, "Stability of differential multipass processes," Electron. Lett., pp. 1016-1016, 1989.

[4] P. P. Khargonekar, I. R. Petersen, and K. Zhou, "Robust stabilization of uncertain linear systems: Quadratic stabilizability and $H_{\infty}$ control theory," IEEE Trans. Automat. Contr., vol. 35, pp. 356-361, Mar. 1990.

[5] D. H. Owens, N. Amann, E. Rogers, and M. C. French, "Analysis of iterative learning control schemes - A 2D systems/repetitive processes approach," Multidim. Syst. Signal Processing, vol. 11, no. 1/2, pp. $125-177,2000$.

[6] P. D. Roberts, "Two-dimensional analysis of an iterative nonlinear optimal control algorithm," IEEE Trans. Circuits Syst. I, vol. 49, pp. 872-878, June 2002.
[7] E. Rogers and D. H. Owens, Stability Analysis for Linear Repetitive Processes. Berlin, Germany: Springer-Verlag, 1992, vol. 175, Lecture Notes in Control And Information Sciences Series.

\section{Comments on "Stability Tests of $N$-Dimensional Discrete Time Systems Using Polynomial Arrays"}

\author{
Li Xu, Jiangqian Ying, Zhiping Lin, and Osami Saito
}

\begin{abstract}
In this brief, we wish to point out that the author of the above paper overlooked a mistake in the stability test procedure for $N$-dimensional $(N-\mathrm{D}, N>2)$ systems proposed in the above paper, which made the polynomial array approach not general. It is shown that Hu's test procedure applies only to a very restricted class of $N$-D stability test problems, and for a general case, instead of necessary and sufficient conditions it provides only sufficient conditions. A counterexample is also given.
\end{abstract}

Index Terms-Multidimensional systems, polynomial array, stability test.

\section{INTRODUCTION AND PROBLEM DESCRIPTION}

The purpose of this brief is to show that the author of [1] overlooked a mistake in the stability test procedure for $N$-dimensional $(N-\mathrm{D}, N>$ 2 ) systems proposed in [1], so that this procedure does not generally serve as a necessary and sufficient condition for $N$-D stability tests except for certain very restricted cases. As the usage of some notations in [1] is a little confusing, we first rephrase the related results of [1] here in a slightly different way.

Consider an $N$-D discrete system described by the transfer function

$$
G\left(z_{1}, \ldots, z_{N}\right)=\frac{P\left(z_{1}, \ldots, z_{N}\right)}{F\left(z_{1}, \ldots, z_{N}\right)}
$$

with $P\left(z_{1}, \ldots, z_{N}\right)$ and $F\left(z_{1}, \ldots, z_{N}\right)$ being $N$-D factor coprime polynomials, and assume that $G\left(z_{1}, \ldots, z_{N}\right)$ possesses no nonessential singularities of the second kind.

The necessary and sufficient condition for $N$-D system (1) to be BIBO stable is that $F\left(z_{N}\right) \triangleq F\left(z_{1}, \ldots, z_{N}\right)$ is devoid of zeros in the closed-unit polydisk, i.e.,

$$
F\left(z_{N}\right) \neq 0, \quad \text { for } \bigcap_{p=1}^{N}\left|z_{p}\right| \leq 1 .
$$

Further, it is well known that this condition is equivalent to a set of tests given by

$$
\begin{aligned}
& F\left(z_{m}\right) \triangleq F\left(z_{1}, \ldots, z_{m}\right) \neq 0, \\
& \quad \text { for } \bigcap_{p=1}^{m-1}\left|z_{p}\right|=1,\left|z_{m}\right| \leq 1, \quad m=1,2, \ldots, N
\end{aligned}
$$

where $F\left(z_{m}\right)$ is obtained by setting $z_{i}=0$ in $F\left(z_{N}\right)$ for $i>m$.

Manuscript received April 1, 2001; revised May 7, 2003. This paper was recommended by Associate Editor D. Mandic.

L. Xu is with the Faculty of System Science and Technology, Akita Prefectural University, Akita 015-0055, Japan (e-mail: xuli@akita-pu.ac.jp).

J. Ying is with the Faculty of Regional Studies, Gifu University, Gifu 501, Japan.

Z. Lin is with the School of Electrical and Electronics Engineering, Nanyang Technological University, Singapore 639798.

O. Saito is with Chiba University, Chiba 263-8522, Japan.

Digital Object Identifier 10.1109/TCSII.2003.816934 